\title{
Standard model atmospheres for A-type stars and non-LTE effects
}

\author{
Jiří Kubát and Daniela Korčáková \\ Astronomický ústav, Akademie věd České republiky, CZ-251 65 Ondřejov, Czech Republic \\ email: kubat@sunstel.asu.cas.cz,kor@sunstel.asu.cas.cz
}

\begin{abstract}
The current status of NLTE model atmosphere calculations of A type stars is reviewed. During the last decade research has concentrated on solving the restricted NLTE line formation problem for trace elements assuming LTE model atmospheres. There is a general lack of calculated NLTE line blanketed model atmospheres for A type stars, despite the availability of powerful methods and computer codes that are able to solve this task. Some directions for future model atmosphere research are suggested.
\end{abstract}

Keywords. Stars: atmospheres, methods: numerical, radiative transfer, stars: early-type

\section{Introduction}

Compared to the atmospheres of hotter $\mathrm{O}$ and $\mathrm{B}$ stars that have stellar winds and to the atmospheres of cooler $\mathrm{F}$ and $\mathrm{G}$ stars that have convective atmospheres, the atmospheres of A-type stars are relatively quiet, which enables the presence of various interesting phenomena. This atmospheric calmness leads to the development of a number of different chemical peculiarities and magnetic field structures on long time scales.

There exist widely used grids of line blanketed LTE ATLAS9 model atmospheres (Kurucz 1993, Castelli \& Kurucz 2003). These models are extensively used for A stars. In addition several attempts to remove the inconsistent assumption of LTE have been performed. The first NLTE calculation of a cool B star, which can be somehow understood as a first step toward A star modelling, was performed by Auer \& Mihalas (1970). The domain of A star temperatures was first investigated by Kudritzki (1973) and Frandsen (1974). A grid of NLTE model atmospheres of stars with $10000 \mathrm{~K} \leqslant T_{\text {eff }} \leqslant 15000 \mathrm{~K}$ (thus also including a part of the A star domain) was calculated by Borsenberger \& Gros (1978). A great improvement was made by Hubený (1980, 1981), who studied in detail the effect of the L $\alpha$ line on the NLTE model atmospheres of A stars and applied his code to Vega. A detailed analysis of the physics needed to replace the assumption of LTE was presented by Hubený (1986). The first NLTE line blanketed model atmospheres of A stars were calculated using the method of superlevels and superlines by Hubeny \& Lanz (1993). Unfortunately, although there has been great progress in calculating NLTE model atmospheres for hot stars and for white dwarfs, calculations of NLTE model atmospheres of A stars are very rare. The only recent NLTE model atmosphere of an A-type star was the model calculated by Hauschildt et al. (1999) for parameters corresponding to Vega. Their announced grid of NLTE models has not appeared.

The inherent reason which stands tacitly behind the lack of calculated NLTE A star model atmospheres is the different scaling properties of the atmospheres of this stellar type. The atmospheres of A stars have very extended line formation regions so that different lines form at very different depths (cf. Fig. 1 in Lanz \& Hubeny 1993). Therefore they are very difficult to describe by a simple standard depth discretization as compared to $\mathrm{O}$ stars and white dwarfs. Hence a much more elaborate depth scale has to be used. Our 
own experience shows that there are severe convergence problems caused by improper depth scaling and only a tremendous number of depth points helped.

\section{Basic equations for NLTE model atmospheres}

A solution of a model atmosphere problem means taking the basic input values of stellar luminosity $L$, stellar radius $R$, and stellar mass $M$ (or, equivalently for the usual plane-parallel case effective temperature $T_{\text {eff }}$ and gravitational acceleration at the stellar surface $g$ ), adding physical laws that are important in the stellar atmosphere (a principal ingredient), and calculating the spatial distribution of various physical quantities (temperature $T(\vec{r})$, electron density $n_{e}(\vec{r})$, population numbers $n_{i}(\vec{r})$, density $\rho(\vec{r})$, velocity field $\vec{v}(\vec{r})$, etc. This procedure is often being performed in substeps. The first step is the determination of $T, n_{e}, \rho$, and $n_{i}$ for the most important opacity sources. The second (optional) step is the determination of $n_{i}$ for the minor abundant species, often referred to as trace elements. The final step in this process is the calculation of the theoretical emergent radiation which may be then compared to the observed one.

A standard model atmosphere here is a static one-dimensional (plane-parallel or spherically symmetric) atmosphere in hydrostatic, radiative, and statistical equilibrium. To calculate such a model atmosphere we need to solve the radiative transfer equation (to determine the radiation field $I$ ), the hydrostatic equilibrium equation (to determine $\rho$ ), the energy equilibrium equation (to determine $T$ ), and the statistical equilibrium equations (to determine $n_{i}$ ).

\subsection{Radiative transfer equation}

The radiative transfer equation is the principal equation for model atmosphere calculations. Regardless of the geometrical approximation which is adopted, the basic solution of the radiative transfer equation is performed along a ray:

$$
\frac{\mathrm{d} I_{\nu}(r)}{\mathrm{d} s}=-\chi_{\nu}(r) I_{\nu}(r)+\eta_{\nu}(r) .
$$

Usually the simplest possibility, i.e., the plane-parallel atmosphere, is being used for the modelling of stellar atmospheres, basically due to its relative simplicity.

\subsubsection{Formal solution}

By formal solution of Equation (2.1) we mean the solution for a given opacity and emissivity. Such a solution may be performed using either differential or integral methods. The latter are especially useful for the multidimensional case. The inconsistency of the formal solution is that opacity $\chi_{\nu}$ and emissivity $\eta_{\nu}$ are not given, but in the process of solving the model atmosphere problem they depend on temperature, density, and radiation field, which are to be determined when solving the radiative transfer equation. However, the formal solution remains at the heart of each model atmosphere code (see Auer 2003).

\subsubsection{Approximate solution using $A L O$}

The process of the formal solution may be expressed using the $\Lambda$-operator

$$
J_{\nu}=\Lambda_{\nu} S_{\nu}
$$

This expression may be used in an iterative process, where the source function $S_{\nu}$ is determined by solving the other constraint equations of hydrostatic, energy, and statistical 
equilibria. As has been shown, such a process, however, effective for optically thin molecular clouds (cf. Dickel \& Auer 1994), fails to produce a convergent solution for optically thick stellar atmospheres (see Auer 1984). This problem has been overcome using the Newton-Raphson method for model stellar atmospheres by Auer \& Mihalas (1969). As an efficient alternative, an approximate $\Lambda$-operator $\Lambda_{\nu}^{*}$ may be used,

$$
J_{\nu}=\Lambda_{\nu}^{*} S_{\nu}+\left(\Lambda_{\nu}-\Lambda_{\nu}^{*}\right) S_{\nu}
$$

The operator $\Lambda_{\nu}^{*}$ is constructed so that it contains the basic physics of the problem and is simply calculable. The most efficient method is to calculate this operator consistently with the numerical method used for the formal solution, as has been described by Rybicki \& Hummer (1991) and by Puls (1991). Then the radiation field is determined by an iterative process,

$$
J_{\nu}^{(n)}=\Lambda_{\nu}^{*}\left[S_{\nu}\left(J_{\nu}^{(n)}\right)\right]+\left(\Lambda_{\nu}-\Lambda_{\nu}^{*}\right)\left[S_{\nu}\left(J_{\nu}^{(n-1)}\right)\right]
$$

where $(n)$ means the current iteration step and $(n-1)$ the previous one.

\subsection{Energy equilibrium}

The energy equilibrium equations determine the temperature structure. They describe the basic energy balance in the atmosphere. For static atmospheres, the equation of radiative equilibrium,

$$
\int_{0}^{\infty}\left(\kappa_{\nu} J_{\nu}-\eta_{\nu}\right) d \nu=\int_{0}^{\infty} \kappa_{\nu}\left(J_{\nu}-S_{\nu}\right) d \nu=0,
$$

which simply describes the radiative energy balance, is usually used. However, this equation does not guarantee radiative flux conservation, which results in an incorrect temperature structure at large optical depths. Therefore, another form, which comes from $\nabla \cdot \overrightarrow{\mathcal{F}}=0$, is used at large optical depths. Better numerical properties of the scheme may be achieved if a linear combination of these equations is considered, as was first done by Hubeny \& Lanz (1995). Another improvement of convergence properties, especially useful at small continuum optical depths where strong lines are still present, was introduced by Kubát et al. (1999) where a thermal balance of electrons instead of the radiative equilibrium equation is used.

Thus three different equations may be used in the stellar atmosphere to take into account energy equilibrium. In the deepest layers, the differential form of radiative equilibrium is used. In the middle parts, where continuum radiation is formed, the integral form of radiative equilibrium works well. In the outer parts where optically thick lines coexist with an optically thin continuum (which is the case of A star atmospheres), the thermal balance of electrons works best. A more detailed discussion with the numerical properties of these methods may be found in Kubát (2003a,b).

\subsection{Equations of statistical equilibrium}

The equations of statistical equilibrium are the key equations both for the NLTE model atmosphere and line formation problems. The set of equations for statistical equilibrium for the static case may be written as

$$
n_{i} \sum_{l}\left(R_{i l}+C_{i l}\right)-\sum_{l} n_{l}\left(R_{l i}+C_{l i}\right)=0
$$

Radiative rates $R_{i l}$ are those responsible for the NLTE effects, since they introduce nonlocal interactions and alter the level population irrespective of local equilibrium conditions. 
They cause the gas not to be in thermodynamic equilibrium. On the other hand, for an equilibrium (Maxwellian) electron velocity distribution (which is the common case), collisional rates reintroduce thermodynamic equilibrium. The balance between collisional and radiative rates determines the applicability of the LTE approximation. If collisions dominate, then the LTE approximation is feasible. If radiative transitions dominate, which is the case in A star atmospheres, then the equations of statistical equilibrium need to be solved to obtain the correct population numbers.

Since the system of rate equations is linearly dependent, we have to close it with some other condition. For a reference (usually, but not necessarily hydrogen) atom either the particle conservation equation $\sum_{k} N_{k}=N-n_{e}$ or the charge conservation equation $\sum_{k} \sum_{j} q_{j} N_{j k}=n_{e}$ is used. For other atoms, the abundance equation $N_{k}=Y_{k} N_{r}$ is usually used. The detailed forms of the equations may be found, e.g., in Kubát (1997).

\subsection{Solution of the system of equations}

The whole system of equations is usually solved using a Newton-Raphson iteration scheme, which in modeling stellar atmospheres used to be referred to as linearization (Auer \& Mihalas 1969). The radiation field is linearized as well, or it can be included using approximate lambda operators (see Eq. 2.4), as was first done by Werner (1986). Excellent reviews of ALI methods of model atmosphere solutions are provided by Hubeny (2003) and Werner et al. (2003). In addition, an implicit linearization of $b$-factors saves additional computer time and memory (Anderson 1987). The whole process may be accelerated using either Kantorovich (see Hubeny \& Lanz 1992) or Ng acceleration (see Auer 1991).

\subsection{Line blanketing}

Line blanketing is caused by the enormous number of spectral lines in the UV region $\left(\sim 10^{7}\right)$, mostly of iron and nickel, which causes radiation to be absorbed in the UV and reemitted in the visible region, thus changing the emergent flux and atmospheric structure significantly. There are two main approaches to line blanketing under the simplifying assumption of LTE, the ODF (Opacity Distribution Function) and OS (Opacity Sampling).

If we do not use the assumption of LTE, we have to solve the equations of statistical equilibrium to obtain the correct population numbers for all levels. This task is relatively simple for simple atoms (like He), but becomes difficult for atoms with a complicated level structure, like iron. To cope with the complexity of these atoms, Anderson (1989) introduced the concept of superlevels and superlines. A superlevel is a level which is created by grouping several individual levels. To achieve a real simplification, it is useful to group the levels in such a manner that the relative population distribution inside the superlevel obeys the LTE distribution. Thus, this grouping is done according to the energy, $E_{i}$, of individual levels (Anderson 1989, Dreizler \& Werner 1993). A more sophisticated grouping according to $E_{i}$ and parity was done by Hubeny \& Lanz (1995). Dreizler \& Werner (1993) used opacity sampling in their calculations, whereas Hubeny \& Lanz (1995) used the NLTE opacity distribution function.

\subsection{LTE versus NLTE}

Since the beginning of NLTE calculations there is a battle between 'LTE people' and 'NLTE people' concerning which approach is better. If NLTE model atmospheres were calculated as easily as the LTE variety, there would be no discussion and everybody would use NLTE. However, this is not the case and it is much more difficult to calculate a NLTE model than the LTE model. Two conflicting aims enter the scene. The first is 
to analyse as many stars as possible, which can be hardly done using NLTE models. The second is to study stars as accurately as possible, which can hardly be done using LTE models.

For the case of LTE it is relatively easy to handle line blanketing, since one neglects the effect of radiation on atomic population numbers, which saves enormous computing time. On the other hand the more general case of NLTE, including line blanketing, is computationally expensive, albeit now it is possible to handle NLTE calculations using sophisticated numerical methods and contemporary computers. However, for extended atmospheres the reliability of LTE decreases and one is forced to use NLTE.

To avoid repeated calculations of complicated model atmospheres one may take the advantage of precomputed grids. However, one has to keep in mind that using such grids, which were calculated under certain physical assumptions, always limit the results by their assumptions.

\section{NLTE line formation calculations}

Calculations of full NLTE model atmospheres of A type stars are a difficult task. Due to a large span of line formation regions down to optical depths in the continuum of about $10^{-9}$, the numerical procedure becomes extremely unstable and the calculations require special care. That is why people started to solve the easier task of NLTE line formation for a given model atmosphere. A necessary condition for such calculation to be reasonable is negligible influence of the ion on the ionization balance and, consequently, on the global structure of the atmosphere. Such elements are being referred to as trace elements.

Contemporary NLTE analysis of A stars are mainly concerned with solving the statistical equilibrium equations for trace elements for a given (mostly LTE) model atmosphere. These calculations have already been reviewed by Hubený (1986) and Hubeny \& Lanz (1993), so only new calculations that appeared after the last review are listed in Table 1. We did our best to mention all published calculations. In the case we unintentionally omitted some, we apologize in advance.

There are several codes available for this purpose. They use slightly different numerical techniques. The basic differences are in the treatment of the radiation field. Both the accelerated lambda iteration and complete linearization methods are used. A comparison of results from different codes for Vega (and Sun) was presented by Kamp et al. (2003), who found large differences in the results of different codes. Therefore, we indicate which code was used.

\section{Beyond standard model atmospheres}

\subsection{Nonthermal collisional rates}

In standard NLTE calculations only the radiation field and level populations are allowed to deviate from their equilibrium values. The velocity distributions of individual species are still assumed to be in equilibrium. Collisional rates are then calculated as an average over the Maxwellian electron velocity distribution,

$$
C_{i j}=n_{e} \int_{v_{0}}^{\infty} \sigma_{i j}(v) f(v) v \mathrm{~d} v .
$$

Thus in equilibrium there is a relation between collisional rates up and down (excitation/deexcitation or ionization/recombination),

$$
n_{i}^{*} C_{i j}=n_{j}^{*} C_{j i}
$$


Table 1. List of NLTE calculations for a given LTE model atmosphere for A stars

\begin{tabular}{|c|c|c|c|}
\hline Ion & Reference & Code & Comment on included levels and transitions \\
\hline He I & Takeda (1994) & 3 & calculations for Deneb; 88 levels \\
\hline $\mathrm{Li}$ I & Mashonkina et al. (2002) & 4 & \\
\hline $\mathrm{Li}$ I/II & Shavrina et al. (2001) & & 20 levels \\
\hline \multirow[t]{4}{*}{$\mathrm{C}_{\mathrm{I}}$} & Takeda (1992b) & 3 & 129 levels, 2351 radiative transitions \\
\hline & Venn (1995) & 2 & 83 levels \\
\hline & Rentzsch-Holm (1996b) & 2 & 83 levels, 63 transitions \\
\hline & Paunzen et al. (1999) & 2 & 83 levels, 63 transitions \\
\hline $\mathrm{C}_{\mathrm{I} / \mathrm{II}}$ & Przybilla et al. (2001b) & 1 & $\mathrm{C}_{\text {I levels with }} n \leqslant 9, \mathrm{C}_{\text {II l levels with } n \leqslant 4}$ \\
\hline \multirow[t]{7}{*}{$\mathrm{NI}^{\prime}$} & Takeda (1992b) & 3 & 119 levels, 2119 radiative transitions \\
\hline & Sadakane et al. 1 (1993) & 3 & used model atom of Takeda (1992b) \\
\hline & Takeda \& Takada-Hidai (1995) & 3 & used model atom of Takeda (1992b) \\
\hline & Takada-Hidai \& Takeda (1996) & 3 & used model atom of Takeda (1992b) \\
\hline & Venn (1995) & 2 & 93 levels, 189 transitions \\
\hline & Lemke \& Venn (1996) & 2 & 93 levels, 189 radiative transitions \\
\hline & Rentzsch-Holm (1996a) & 2 & 96 levels, 82 transitions \\
\hline $\mathrm{NI} / \mathrm{II}$ & Przybilla \& Butler (2001) & 1 & $\mathrm{~N}_{\text {I levels }}$ with $n \leqslant 7, \mathrm{~N}_{\text {II }}$ levels with $n \leqslant 6$ \\
\hline \multirow[t]{5}{*}{ O I } & Takeda (1992a, 1997) & 3 & 86 levels, 294 radiative transitions \\
\hline & Takeda \& Takada-Hidai (1998) & 3 & 86 levels, 294 radiative transitions \\
\hline & Takeda et al. (1999) & 3 & calculations from Takeda (1997) for CP stars \\
\hline & Paunzen et al. (1999) & 2 & 15 levels \\
\hline & Przybilla et al. (2000) & 1 & all levels below excitation \\
\hline \multirow[t]{2}{*}{$\mathrm{NaI}$} & Takeda \& Takada-Hidai (1994) & 3 & $\begin{array}{l}\text { calculations for A supergiants, } 92 \text { levels, } 178 \\
\text { radiative transitions }\end{array}$ \\
\hline & Mashonkina et al. (2000) & 4 & solution for $T_{\text {eff }}=4000-12000 \mathrm{~K}, 21$ levels \\
\hline \multirow[t]{2}{*}{ Mg I } & Shimanskaya et al. (2000) & 4 & solution for $T_{\text {eff }}=4500-12000 \mathrm{~K}, 50$ levels \\
\hline & Idiart \& Thévenin (2000) & 5 & 104 levels, 980 radiative transitions \\
\hline $\mathrm{Mg} \mathrm{I} / \mathrm{II}$ & Przybilla et al. (2001a) & 1 & all levels with $n \leqslant 10$ \\
\hline \multirow[t]{2}{*}{$\mathrm{S}$ I } & Takeda \& Takada-Hidai (1995) & 3 & 56 levels, 173 radiative transitions \\
\hline & Takada-Hidai \& Takeda (1996) & 3 & 56 levels, 173 radiative transitions \\
\hline $\mathrm{K} \mathrm{I}$ & Ivanova \& Shimanskii (2000) & 4 & solution for $T_{\text {eff }}=4000-10000 \mathrm{~K}, 36$ levels \\
\hline $\mathrm{Ca} \mathrm{I}$ & Idiart \& Thévenin (2000) & 5 & 84 levels, 483 transitions \\
\hline Ti II & Becker (1998) & 1 & complete model atom, using \\
\hline Fe II & Becker (1998) & 1 & complete model atom, using superlevels \\
\hline \multirow[t]{2}{*}{$\mathrm{Fe} \mathrm{I} / \mathrm{II}$} & Rentzsch-Holm (1996b) & 2 & $79+20$ levels, $52+23$ lines \\
\hline & Thévenin \& Idiart (1999) & 5 & $256+190$ levels, $2117+3443$ lines \\
\hline Sr II & Belyakova et al. (1999) & 4 & solution for $T_{\text {eff }}=4000-12000 \mathrm{~K}, 41$ levels \\
\hline Nd II/III & Mashonkina et al. (2005) & 1 & $247+69$ levels \\
\hline
\end{tabular}

Computer codes used : 1 - DETAIL (Giddings 1981); 2 - Kiel (Steenbock \& Holweger 1984); 3 - Takeda (1991); 4 - NONLTE3 (Sakhibullin 1983); 5 - MULTI (Carlsson 1986).

If the velocity distribution is non-Maxwellian (e.g., in electron beams formed in the Sun during flares - cf. Kašparová \& Heinzel 2002), then equation (4.2), which expresses the equilibrium condition, is invalid. In such a case the collisions may cause level population numbers to differ from the LTE values, which has an observable effect on the line profiles. The nonthermal collisional term may be considered as another source of NLTE effects. Such effects are present in the Sun, where electron beams are formed after magnetic reconnection. There is also a possibility that they may be present in magnetic A stars, where releasing magnetic energy in eruptive events like flares may be expected as well.

\subsection{Magnetic fields}

Inclusion of magnetic fields into model atmosphere calculations have been done only occasionally. An important attempt in calculating a model atmosphere with a magnetic field was done by Carpenter (1985), who found changes in the net gravity and pressure 
distribution due to the magnetic field. The most consistent model so far was recently developed by Valyavin et al. (2004), who assumed LTE and included the Lorentz force into the equation of hydrostatic equilibrium. A historical overview of magnetic model atmosphere calculations is also presented there.

\subsection{Diffusion}

An important aspect of A star atmospheres is that atmospheres of a good fraction of stars of this stellar spectral type are extremely quiet. Such quiet atmospheres without significant global motions such as global convection and stellar winds enable long time scale processes of diffusion and gravitational settling to take place. Diffusion occurs not only in the presence of magnetic field (note the effect of enhancing radiative acceleration in a polarized radiation field and the effect of ambipolar diffusion, Babel \& Michaud (1991a, 1991b), but also due to different sensitivities of various atoms and ions to incoming radiation. Radiative diffusion is able to explain various chemical peculiarities in $\mathrm{CP}$ stars and is also responsible for the isotopic shift effect (Aret \& Sapar 2002). However, consistent NLTE model atmospheres that take into account radiative diffusion processes are still missing for A type stars. NLTE model atmospheres with diffusion were calculated by Dreizler \& Shuh (2003) for white dwarfs, where diffusion processes are also important. The physical process of diffusion is reviewed in detail by Michaud (2005).

\subsection{Extended atmospheres}

Plane-parallel model atmospheres are also being used for NLTE modeling of atmospheres of A-type supergiants (e.g., Kudritzki 1973). However, they do not describe the limb darkening correctly. A-type supergiants have also stellar winds and show P Cyg line profiles. Therefore it is impossible to describe them using plane parallel atmospheres. Also, wind models of A stars are not calculated very often. Multicomponent hydrodynamic radiatively driven wind models for A stars were calculated by Babel (1995). Another radiation driven wind model was calculated by Achmad et al. (1997), but they did not take into account for NLTE effects. Generally, for extended atmospheres NLTE effects are stronger. An attempt to calculate spherically symmetric NLTE model atmospheres with a stellar wind for Deneb was done by Aufdenberg et al. (2002). However, as the authors note, they were not able to fit the observed $\mathrm{H} \alpha$ line profile. Work on developing consistent NLTE wind models of A supergiants is currently in progress (Krtička \& Kubát 2005).

\subsection{Limb darkening}

An important property of the emergent radiation from stellar atmospheres is the angular dependence of the specific intensity $I(\theta)$, usually called limb darkening. Approximate limb darkening laws are often used (see, e.g., Allen 1963 or Gray 1976). These approximate laws do not describe the angular dependence of the specific intensity very well even in the case of thin stellar atmospheres. In addition, limb darkening is strongly frequency dependent (Hadrava \& Kubát 2003), and in the center of a line, even limb brightening instead of darkening may appear, as can be seen in Figure 1. This effect becomes very strong especially for extended stellar atmospheres.

For a correct description of limb darkening it is necessary to use model atmospheres with a better geometry than plane-parallel. This has been done by Claret \& Hauschildt (2003), who, using a spherically symmetric code, calculated limb darkening for a grid of model atmospheres from $\mathrm{A}$ to $\mathrm{G}$ spectral types.

Knowledge of an accurate limb darkening law is necessary for the detection of stellar spots (see Kjurkchieva 1989). It is also very important for the analysis of interferometric measurements, as well as for the correct treatment of stellar rotation. Fortunately, 

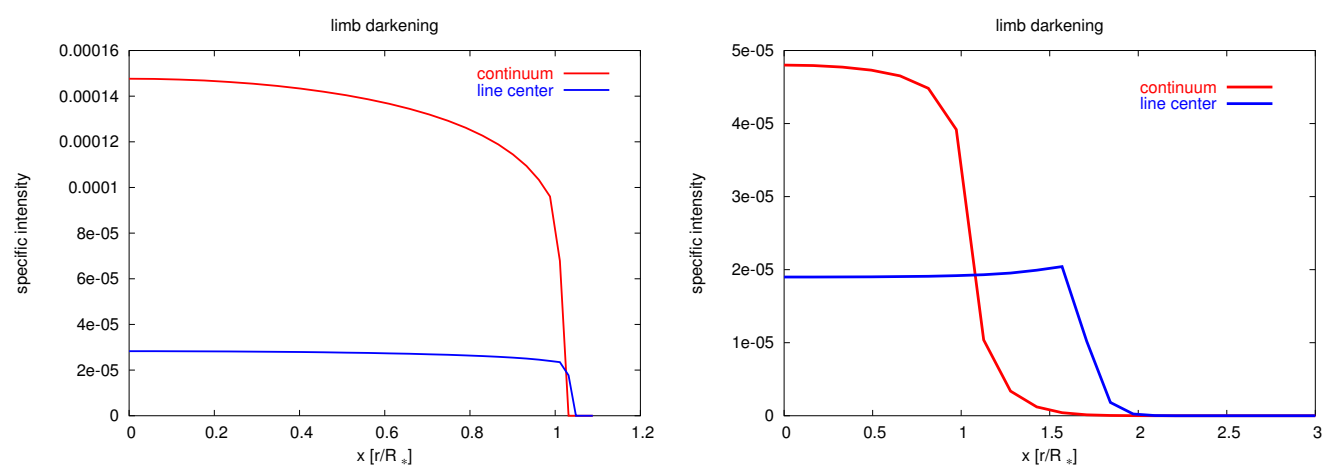

Figure 1. The comparison of limb darkening for the thin atmosphere (left panel) and the extended atmosphere (right panel). These results were obtained from the Korčáková \& Kubát (2003) code.

eclipses from binaries (e.g., Twigg 1979) and gravitational microlensing (Heyrovský 2003) measurements of limb darkening are possible for at least some distant stars.

\subsection{Rotation}

Stellar rotation is usually being accounted by convolving the rotation profile with the static line profile (see Gray 1976). This technique is inaccurate not only due to using only approximate limb darkening laws, but also because the dependence of limb darkening on frequency as well as gravity darkening are neglected. A more accurate method is to integrate the static intensity over the rotating disk of the star, where gravity darkening can also be included. A "simple" way of including gravity darkening in this integration is described by Collins (1963). This method was used by Gulliver et al. (1994) to determine the rotation of Vega.

Stellar rotation is not like rigid body rotation, as it is usually assumed, but it is probably differential. Until now, there are only few measurements of differential rotation. Unfortunately, in many investigations, the authors use the simplest form of the limb darkening law and their results can be used only with reservations.

For an exact description of stellar rotation, plane-parallel model atmospheres are insufficient. It is necessary to use a hydrodynamic multidimensional model atmosphere, at least an axially symmetric one together with the correct description of the radiation field. Recently, we started to work on such a model (Korčáková et al. 2005).

\section{Conclusions}

Full NLTE model atmospheres of A stars are difficult to calculate, since the lines of different ions can form at very different depths, and, therefore, there is a need for a huge number of depth points to resolve all line formation regions sufficiently. Only LTE model atmospheres together with NLTE calculations for individual ions have been calculated in significant numbers. This underlines the need for more intensive calculations of NLTE model atmospheres of A stars. Adding consistently new different physical processes to NLTE model atmospheres calculations, such as diffusion (see Michaud 2005), magnetic fields (see Moss 2005), and more accurate treatments of convection (see Kupka 2005), are necessary. This is a real challenging task for the near future.

\section{Acknowledgements}

The authors would like to thank Dr. Adéla Kawka for her comments on the manuscript. This research has made an extensive use of the ADS. This work was supported by 
grants GA ČR 205/02/0445, 205/04/P224, and 205/04/1267. The Astronomical Institute Ondřejov is supported by projects K2043105 and Z1003909.

\section{References}

Achmad, L., Lamers, H. J. G. L. M., Pasquini, L. 1997, A\&A 320, 196

Allen, C. W. 1963, Astrophysical Quantities, University of London, The Athlone Press

Anderson, L. S. 1987, in W. Kalkofen (ed.), Numerical Radiative Transfer, Cambridge Univ. Press, p. 163

Anderson, L. S. 1989, ApJ 339, 558

Aret, A. \& Sapar, A. 2002, AN 323, 21

Auer, L. H. 1984, in W. Kalkofen (ed.), Methods in Radiative Transfer, Cambridge Univ. Press., p. 237

Auer, L. H. 1991, in L. Crivellari, I. Hubený, \& D. G. Hummer (eds.), Stellar Atmospheres: Beyond Classical Models, NATO ASI Series C 341, Kluwer Academic Publishers, p. 9

Auer, L. H. 2003, ASPC 288, 3

Auer, L. H. \& Mihalas, D. 1969, ApJ 158, 641

Auer, L. H. \& Mihalas, D. 1970, ApJ 160, 233

Aufdenberg, J. P., Hauschildt, P. H., Baron E., et al. 2002, ApJ 570, 344

Babel, J. 1995, A\&A 301, 823

Babel, J. \& Michaud, G. 1991a, A\&A 241, 493

Babel, J. \& Michaud, G. 1991b, A\&\&A 248,155

Becker, S. R. 1998, in I. Howarth (ed.), Boulder-Munich II: Properties of Hot, Luminous Stars, ASP Conf. Ser. Vol. 120, Astron. Soc. Pacific, San Francisco, p. 137

Belyakova, E. V., Mashonkina, L. I., Sakhibullin, N. A. 1999, Astron. Zh. 76, 929

Borsenberger, J. \& Gros, M. 1978, A\&SAS 31, 291

Carlsson, M. 1986, Uppsala Astronom. Obs. Rep. 33

Carpenter, K. G. 1985, ApJ 289, 660

Castelli, F. \& Kurucz, R. L. 2003, IAUS 210, A20

Claret, A. \& Hauschildt, P. H. 2003, A\& A 412, 241

Collins, G., W. 1963, ApJ 138, 1134

Dickel, H. R. \& Auer, L. H. 1994, ApJ 437, 222

Dreizler, S. \& Schuh, S. 2003, IAUS 210, p. 33

Dreizler, S. \& Werner, K. 1993, A\&A 278, 199

Frandsen, S. 1974, A\&A 37, 139

Giddings, J. 1981, PhD thesis

Gray, D. F. 1976, Observation and Analysis of Stellar Photospheres, John Wiley \& Sons, New York

Gulliver, A. F., Hill, G., Adelman, S. J., 1994, ApJ 429, 81

Hadrava, P. \& Kubát, J. 2003, ASPC 288, 149

Heyrovský, D. 2003, ApJ 594, 464

Hauschildt, P. H., Allard, F., Baron, E. 1999, ApJ 512, 377

Hubený, I. 1980, A\&A 86, 225

Hubený, I. 1981, A\&A 98, 96

Hubený, I. 1986, in C. R. Cowley, M. M. Dworetsky \& C. Mégessier (eds.), Upper Main Sequence Stars with Anomalous Abundances, IAU Coll. 90, D. Reidel Publ. Comp., Dordrecht, p. 57

Hubeny, I. 2003, ASPC 288, 17

Hubeny, I. \& Lanz, T. 1992, A\&A 262, 501

Hubeny, I. \& Lanz, T. 1993, ASPC 44, 98

Hubeny, I. \& Lanz, T. 1995, ApJ 439, 875

Hubeny, I., Hummer, D. G., Lanz, T. 1994, A\&A 282, 151

Idiart, T. \& Thévenin, F. 2000, ApJ 541, 207

Ivanova, D. V., Shimanskii, V. V. 2000, Astron. Zh. 77, 447

Kamp. I., Korotin, S., Mashonkina, L., Przybilla, N., Shimansky, S. 2003, IAUS 210, p. 323 
Kašparová, J. \& Heinzel, P. 2002, A $\mathscr{\xi} A$ 382, 688

Kjurkchieva, D. P. 1989, Ap\&্SSS 159, 333

Korčáková, D., Kubát J. \& D.,Krtička, J. 2005, These Proceedings, AP4

Korčáková, D., \& Kubát J. 2003, IAUS 210, B8

Krtička, J. \& Kubát J. 2005, These Proceedings, 23

Kubát, J. 1997, A\& A 326, 277

Kubát, J. 2003a, ASPC 288, 91

Kubát, J. 2003b, IAUS 210, A8

Kubát, J., Puls, J., Pauldrach, A. 1999, A\&\&A 341, 587

Kudritzki, R.-P. 1973, A\&A 28, 103

Kupka, F. 2005, These Proceedings, 119

Kurucz, R. L. 1993, Solar Abundance Model Atmospheres, Kurucz CD-ROM No.19

Lanz, T., \& Hubeny, I. 1993, ASPC 44, 517

Lemke, M. \& Venn, K. A. 1996, A\&A 309, 558

Mashonkina, L. I., Shimanskii, V. V., Sakhibullin, N. A. 2000, Astron. Zh. 77, 893

Mashonkina, L. I., Shavrina, A., Khalack, V., et al. 2002, Astron. Zh. 79, 31

Mashonkina, L. I., Ryabchikova, T. A., Ryabtsev, A. N. 2005, These Proceedings, 315

Moss, D. 2005, These Proceedings, 245

Michaud, G. 2005, These Proceedings, 173

Paunzen, E., Kamp, I., Iliev, I. K., et al. 1999, A\&A 345, 597

Przybilla, N. \& Butler, K. 2001, A\&̈A 379, 955

Przybilla, N., Butler, K., Becker, S. R., Kudritzki, R. P., Venn, K. A. 2000, A\&GA 359, 1085

Przybilla, N., Butler, K., Becker, S. R., Kudritzki, R. P. 2001a, A\&\&A 369, 1009

Przybilla, N., Butler, K., Kudritzki, R. P. 2001b, A\& A 379, 936

Puls, J. 1991, A\&A 248, 581

Rentzsch-Holm, I. 1996a, A\&A 305, 275

Rentzsch-Holm, I. 1996b, $A \& A$ A 312, 966

Rybicki, G. B. \& Hummer, D. G. 1991, A\&A 245, 171

Sadakane, K., Takeda, Y., Okyudo, M. 1993, PASJ 45, 471

Sakhibullin, N. A. 1983, Trudy Kaz. Obs. 48, 9

Shavrina, A. V., Polosukhina, N. S., Zverko, J., et al. 2001, A\&A 372, 571

Shimanskaya, N. N., Mashonkina, L. I., Sakhibullin, N. A. 2000, Astron. Zh. 77, 599

Steenbock, W. \& Holweger, H. 1984, A\& A 130, 319

Takada-Hidai, M. \& Takeda, Y. 1996, PASJ 48, 739

Takeda, Y. 1991, A\&A 242, 455

Takeda, Y. 1992a, PASJ 44, 309

Takeda, Y. 1992b, PASJ 44, 649

Takeda, Y. 1994, PASJ 46, 181

Takeda, Y. 1997, PASJ 49, 471

Takeda, Y. \& Takada-Hidai, M. 1994, PASJ 46, 395

Takeda, Y. \& Takada-Hidai, M. 1995, PASJ 47, 169

Takeda, Y. \& Takada-Hidai, M. 1998, PASJ 50, 629

Takeda, Y., Takada-Hidai, M., Jugaku, J., Sakaue, A., \& Sadakane, K. 1999, PASJ 51, 961

Thévenin, F. \& Idiart, T. 1999, ApJ 521, 753

Twigg, L. W. 1979, MNRAS 189, 907

Venn, K. A., 1995, ApJ 449, 839

Valyavin, G., Kochukhov, O., Piskunov, N. 2004, A\&A 420, 993

Werner, K. 1986, A\& A 161, 177

Werner, K., Deetjen, J. L., Dreizler, S., et al. 2003, ASPC 288, 31 